\title{
Triterpenoids and Semisynthetic Derivatives with Antimicrobial Activities from the Leaves of Caloncoba glauca (Flacourtiaceae)
}

\author{
James D. Simo Mpetga1 ${ }^{*}$, Arno R. Nanfack Donfack1, Jean-De-Dieu Tamokou², \\ Irene Chinda Kengne ${ }^{2}$, Pierre Tane ${ }^{1 \#,}$ Xiao-Jiang Hao ${ }^{3}$, Mathieu Tene ${ }^{1 *}$ \\ ${ }^{1}$ Natural Products Chemistry Research Unit, Department of Chemistry, Faculty of Science, University of Dschang, \\ Dschang, Cameroon \\ ${ }^{2}$ Research Unit of Microbiology and Antimicrobial Substances, Department of Biochemistry, Faculty of Science, \\ University of Dschang, Dschang, Cameroon \\ ${ }^{3}$ State Key Laboratory of Phytochemistry and Plant Resources in West China, Kunming Institute of Botany, \\ Chinese Academy of Sciences, Kunming, China \\ Email: *james.mpetga@univ-dschang.org, *mtene2001@yahoo.fr
}

How to cite this paper: Mpetga, J.D.S., Donfack, A.R.N., Tamokou, J.-D.-D., Kengne, I.C., Tane, P., Hao, X.-J. and Tene, M. (2021) Triterpenoids and Semisynthetic Derivatives with Antimicrobial Activities from the Leaves of Caloncoba glauca (Flacourtiaceae). Advances in Biological Chemistry, 11, 149164

https://doi.org/10.4236/abc.2021.114011

Received: June 10, 2021

Accepted: July 20, 2021

Published: July 23, 2021

Copyright $\odot 2021$ by author(s) and Scientific Research Publishing Inc. This work is licensed under the Creative Commons Attribution International License (CC BY 4.0).

http://creativecommons.org/licenses/by/4.0/

\section{(c) (i) Open Access}

\begin{abstract}
Chemical investigation of the $\mathrm{MeOH}$ extract from the leaves of $C$. glauca yielded nine known triterpenoids (1-9) belonging to the cycloartane and friedelane series. Two of these compounds namely glaucartanoic acid A (1) and $3 \beta, 21 \beta$-dihydroxy-30-nor-(D:A)-friedoolean-20(29)-en-27-oic acid (5) were subjected to chemical derivatizations and afforded five new derivatives: diacetylglaucartanoic acid A (1a), 24-acetylglaucartanoic acid A (1b), glaucartanoic acid A methyl ester (1c), 24-methoxyglaucartanoic acid A methyl ester (1d), and 3 $\beta, 21 \beta$-diacetoxy-30-nor-(D:A)-friedoolean-20(29)-en-27-oic acid (5a). Their structures were assigned based on their NMR and MS data and by comparison with literature values. The $\mathrm{MeOH}$ extract, isolated compounds and some new semi-synthetic derivatives were subjected to in vitro antimicrobial assays against a panel of pathogenic microorganisms, including Gram-positive and Gram-negative bacteria, and fungi using broth microdilution method. The $\mathrm{MeOH}$ extract displayed activity towards all the tested pathogenic bacterial and fungal strains with good activity (MIC $<100 \mu \mathrm{g} / \mathrm{mL}$ ) against Staphylococcus aureus ATCC25923 and Shigella flexneri SDINT. Compounds 3 and 5 showed the most potent antimicrobial effect.
\end{abstract}

\section{Keywords}

Caloncoba glauca, Triterpenoids, Chemical Derivatizations, Antimicrobial 
Activity, Structure-Activity Relationship

\section{Introduction}

Antimicrobial resistance has emerged as one of the major public health concerns of the $21^{\text {st }}$ century that threatens the effective prevention and treatment of an ever-increasing range of infections caused by bacteria, parasites, viruses and fungi that are no longer susceptible to the common medicines used for their treatment [1]. The rapid emergence of resistant microorganisms is occurring worldwide, undermining many other advances in health and medicine. The situation is most dire in less developed countries, where the uncontrolled use of antibiotics, often coupled with a lack of proper healthcare infrastructure, leads to high morbidity rates [2]. Thus, the need of searching and developing new therapeutic agents capable of overcoming the resistance mechanisms of microbes is imperative [3], and plants with medicinal properties constitute a rich source of biologically active compounds. Triterpenoids, a class of compounds widely distributed in nature have shown diverse biological activities, including antimicrobial properties [4] [5]. Structure-activity studies have revealed that alterations in key carbons may enhance biological activity and reduce cytotoxicity [6]. Previous chemical studies of some Caloncoba species have proved this genus to be a rich source of triterpenoids [7]-[15]. Caloncoba glauca (P. Beauv.) Gilg (Flacourtiaceae) is a tree up to $15 \mathrm{~m}$ tall found in tropical Africa [16]. Its leaves are used as purgative and against inflammations and skin diseases [11]. Many microbial species including Staphylococcus aureus, Pseudomonas aeruginosa, Candida tropicalis, Candida albicans and Cryptococcus neoformans are capable of causing various skin and other organs infections. Skin lesions due to $C$. neoformans are present in almost $5 \%$ of patients with cryptococcal meningitis [17] and the frequency is greater in liver transplant recipients receiving tacrolimus [18] or in patients infected with serotype D [19]. In most cases, skin lesions are due to hematogenous spread (i.e., secondary cutaneous cryptococcosis). This prompted us, in connection with our search for bioactive compounds [13] [20] [21] [22], to reinvestigate the leaves of C. glauca in view of evaluating its antimicrobial properties. In the present paper, we describe the structure elucidation of five new semi-synthetic cycloartanes $(1 \mathrm{a}$ 1d) and friedelane-type (5a) triterpenoids obtained from the chemical transformations of two out of the nine triterpenoids isolated, and the antimicrobial activity of both the naturally occurring triterpenoids and the semi-synthetic derivatives. The structure-activity relationship is also discussed.

\section{Material and Methods}

\subsection{Plant Material}

C. glauca was selected on the basis of its utilization in traditional medicine. Its leaves were harvested in Bangang-Wabane village, Cameroon, in May 2016. The 
sample was authenticated by Mr. Francois NANA, a botanist of the National Herbarium of Cameroon (Yaounde, Cameroon), where a voucher specimen (55064/HNC) was deposited.

\subsection{Extraction and Isolation}

The air-dried and powdered leaves of $C$. glauca $(1.2 \mathrm{~kg}$ ) were extracted with methanol $(3 \times 10 \mathrm{~L})$ for $72 \mathrm{~h}$ at room temperature to yield a crude extract ( $90 \mathrm{~g})$ after evaporation of solvent. Part of this extract (85 g) was subjected to column chromatography $(8 \mathrm{~cm} \times 60 \mathrm{~cm})$ over silica gel $(300-400$ mesh) eluted with gradients of $n$-Hex/EtOAc (90:10, 70:30, 50:50, 30:70) then EtOAc/MeOH $(100: 0,90: 10,70: 30,50: 50,0: 100)$ and 68 fractions of $300 \mathrm{~mL}$ each were collected. These fractions were combined on the basis of their TLC (Thin Layer Chromatography) profiles into five major fractions A-E: A (9 g, 1 - 14), B (14 g, 15 - 28), C (16 g, 29 - 43), D (12 g, 44 - 53) and E (23 g, 54 - 68). Fraction A with a fatty appearance was not further investigated. Fraction B (14 g) was separated on a silica gel (100 - 200 mesh) column chromatography using a gradient of $n-\mathrm{Hex} / \mathrm{Me}_{2} \mathrm{CO}$ (100:0 to $70: 30)$ to yield caloncobalactone C (8, $12 \mathrm{mg})$. Fraction C (16 g) was separated on a silica gel (100 - 200 mesh) column eluted with gradients of $n$ $\mathrm{Hex} / \mathrm{EtOAc}(90: 10$ to $0: 100)$ then EtOAc/MeOH (95:5 to 0:100) and subfractions C.I to C.IV were obtained. Successive column chromatography of subfraction C.I (2.5 g) over silica gel (100 - 200 mesh) and Sephadex LH-20 eluted respectively with gradients of $n-\mathrm{Hex} / \mathrm{Me}_{2} \mathrm{CO}$ (90:10 to 100:0) and isocratic $\mathrm{CH}_{2} \mathrm{Cl}_{2} /$ $\mathrm{MeOH}$ (1:1) solvents mixtures afforded glaucartanoic acid B (4, $9.5 \mathrm{mg})$. Repeated purifications of sub fraction C.II ( $4 \mathrm{~g})$ on silica gel (100 - $200 \mathrm{mesh}$ ) column chromatography eluted with $\mathrm{CH}_{2} \mathrm{Cl}_{2} / \mathrm{Me}_{2} \mathrm{CO}$ (100:0 to 50:50) afforded caloncobic acid A (6, $14 \mathrm{mg})$. Subfraction C.III (3.5 g) was subjected successively to a silica gel (100 - 200 mesh) column chromatography eluted with $\mathrm{CH}_{2} \mathrm{Cl}_{2} /$ $\mathrm{Me}_{2} \mathrm{CO}\left(100: 0\right.$ to 70:30) and passage over Sephadex LH-20 $\left(\mathrm{CH}_{2} \mathrm{Cl}_{2} / \mathrm{MeOH}, 1: 1\right)$ to give glaucalactone $(3,19 \mathrm{mg})$. Sub fraction C.IV $(1.5 \mathrm{~g})$ precipitated in $n$-Hex/ $\mathrm{Me}_{2} \mathrm{CO}$ (80:20) and the deriving powder was purified over a Sephadex LH-20 column chromatography eluted with $\mathrm{CH}_{2} \mathrm{Cl}_{2} / \mathrm{MeOH},(1: 1)$ to afford glaucalactone B (9, $8.4 \mathrm{mg})$. Fraction D (12 g) was chromatographed on a silica gel (100 $200 \mathrm{mesh})$ column eluted with gradients of $n$-Hex/EtOAc (90:10 to 0:100) then EtOAc/MeOH (95:5 to 0:100) to give three subfractions: D.I, D.II and D.III. Subfraction D.II (7 g) was subjected to a silica gel (100 - 200 mesh) column chromatography eluted with $n-\mathrm{Hex} / \mathrm{Me}_{2} \mathrm{CO}$ (from 90:10 to 0:100) and afforded sub fractions D.II.1 - D.II.3. Successive chromatography of subfraction D.II.2 (2.3 g) over a silica gel column eluted with $n-\mathrm{Hex} / \mathrm{Me}_{2} \mathrm{CO}(70: 30$ to $100: 0)$ and Sephadex LH-20 eluted with $\mathrm{CH}_{2} \mathrm{Cl}_{2} / \mathrm{MeOH}$ (1:1) yielded glaucatarnoic acid A (1, $170 \mathrm{mg}$ ) and 3 $\beta, 21 \beta$-dihydroxy-30-nor-(D:A)-friedoolean-20(29)-en-27-oic acid $(5,30 \mathrm{mg})$. Subfraction D.II.3 (1.8 g) was purified similarly to D.II.2 to furnish caloncobalactone $(2,14.3 \mathrm{mg})$. Fraction E (23 g) precipitated in EtOAc. Purification of the filtered powder over a silica gel column chromatography eluted with EtOAc/MeOH (95:5) followed by gel permeation over a Sephadex LH-20 
column using $\mathrm{CH}_{2} \mathrm{Cl}_{2} / \mathrm{MeOH}$, (1:1) gave caloncobic acid C (7, $\left.8.3 \mathrm{mg}\right)$.

\subsection{Chemical Transformations and Purifications}

\subsubsection{Acetylation of Glaucartarnoic Acid A (1)}

A sample (15 mg, $30.7 \mathrm{mmol}$ ) of glaucartanoic acid A (1) was dissolved in pyridine- $\mathrm{Ac}_{2} \mathrm{O}(3 \mathrm{ml}, 1: 1)$. Catalytic amount of 4-DMAP was added and the mixture was kept overnight in darkness at room temperature (Scheme 1). The reaction mixture was poured in cold water at $5^{\circ} \mathrm{C}$ and extracted with EtOAc. The organic layer was washed with $2 \mathrm{M} \mathrm{HCl}$, then $1 \mathrm{M} \mathrm{NaHCO}_{3}$ and dried using a rotary evaporator. Column chromatography over silica gel ( $\left.n-\mathrm{Hex} / \mathrm{Me}_{2} \mathrm{CO}, 9: 1\right)$ gave 24-acetylglaucartanoic acid A (1b, $12.7 \mathrm{mg}, 80 \%)$.

This reaction was repeated with the same amount of reactants but was heated at $60^{\circ} \mathrm{C}$ for 4 hours (Scheme 1). The reaction mixture worked-up in the same conditions yielded diacetylglaucartanoic acid A (1a, $14.3 \mathrm{mg}, 81 \%)$ and a very little amount of $\mathbf{1 b}$.

Diacetylglaucartanoic acid A (1a): White powder (from $\mathrm{Me}_{2} \mathrm{CO}$ ); $[\alpha]_{\mathrm{D}}^{23}-$ 49.4 (c 0.11, MeOH); ${ }^{1} \mathrm{H}\left(400 \mathrm{MHz}, \mathrm{CD}_{3} \mathrm{OD}\right)$ and ${ }^{13} \mathrm{C}$ (100 MHz, $\left.\mathrm{CD}_{3} \mathrm{OD}\right)$ NMR data, see Table 1 and Table 2 respectively; positive ESIMS m/z (rel. int.) 596 [M $+\mathrm{H}+\mathrm{Na}]^{+}(6), 573[\mathrm{M}+\mathrm{H}]^{+}(5), 513[\mathrm{M}-\mathrm{AcO}]^{+}(100), 453[\mathrm{M}-\mathrm{H}-2 \mathrm{AcO}]^{+}$ (5); HRESIMS m/z $595.3612[\mathrm{M}+\mathrm{Na}]^{+}$(calcd for $\mathrm{C}_{34} \mathrm{H}_{52} \mathrm{O}_{7} \mathrm{Na}, 595.3610$ ).

24-acetylglaucartanoic acid A (1b): White powder (from $\mathrm{Me}_{2} \mathrm{CO}$ ); $[\alpha]_{\mathrm{D}}^{23}-$ 36.9 ( c 0.12, $\mathrm{MeOH}) ;{ }^{1} \mathrm{H}\left(400 \mathrm{MHz}, \mathrm{CD}_{3} \mathrm{OD}\right)$ and ${ }^{13} \mathrm{C}\left(100 \mathrm{MHz}, \mathrm{CD}_{3} \mathrm{OD}\right) \mathrm{NMR}$ data, see Table 1 and Table 2 respectively; positive ESIMS m/z (rel. int.) 531 [M $+\mathrm{H}^{+}(5), 553[\mathrm{M}+\mathrm{Na}]^{+}(8), 513\left[\mathrm{M}+\mathrm{H}-\mathrm{H}_{2} \mathrm{O}\right]^{+}(100) ;$ HRESIMS m/z $553.3505[\mathrm{M}+\mathrm{Na}]^{+}$(calcd for $\left.\mathrm{C}_{32} \mathrm{H}_{50} \mathrm{O}_{6} \mathrm{Na}, 553.3505\right)$.

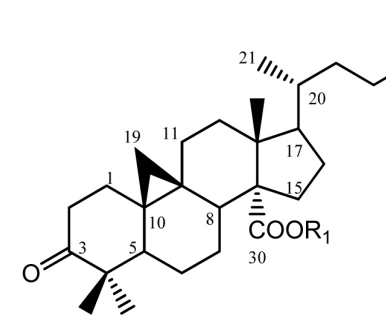

1: $\quad \frac{\underline{\mathbf{R} 1}}{\mathrm{H}} \quad \frac{\mathbf{R 2}}{\mathrm{H}} \quad \frac{\mathbf{R 3}}{\mathrm{H}}$



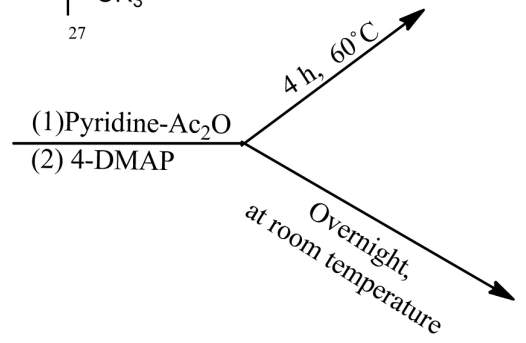

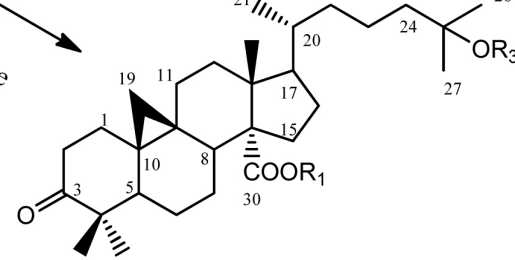

1b: $\frac{\underline{\mathbf{R} 1}}{\mathrm{H}} \quad \frac{\mathbf{R 2}}{\mathrm{Ac}} \quad \frac{\mathbf{R 3}}{\mathrm{H}}$

Scheme 1. Semisynthesis of $1 \mathrm{a}$ and $1 \mathrm{~b}$ from glaucartanoic acid A (1). 
Table 1. ${ }^{1} \mathrm{H}-\mathrm{NMR}$ data of compounds $1 \mathrm{a}-1 \mathrm{~d}$ and $5 \mathrm{a}$.

\begin{tabular}{|c|c|c|c|c|c|}
\hline Position & $1 \mathrm{a}^{*}($ in $\mathrm{MeOD})$ & $1 b^{*}($ in $\mathrm{MeOD})$ & $1 c^{* *}($ in $\mathrm{MeOD})$ & $1 \mathrm{~d}^{* *}($ in $\mathrm{MeOD})$ & $5 \mathrm{a}^{*}\left(\right.$ in $\left.\mathrm{CDCl}_{3}-\mathrm{MeOD}\right)$ \\
\hline 1 & $1.89 \mathrm{~m} ; 1.66 \mathrm{~m}$ & $1.85 \mathrm{~m} ; 1.66 \mathrm{~m}$ & $1.87^{\mathrm{ov}} ; 1.66^{\mathrm{ov}}$ & $1.89 \mathrm{~m} ; 1.66 \mathrm{~m}$ & $1.42^{\mathrm{ov}}(2 \mathrm{H})$ \\
\hline 2 & $\begin{array}{c}2.24 \mathrm{~m} ; \\
2.79 \mathrm{td}(13.8,6.4)\end{array}$ & $\begin{array}{c}2.24 \mathrm{~m} ; \\
2.79 \mathrm{td}(13.8,6.4)\end{array}$ & $\begin{array}{c}2.26 \mathrm{~m} ; \\
2.80 \operatorname{td}(13.9,6.3)\end{array}$ & $\begin{array}{c}2.23 \mathrm{~m} ; \\
2.80 \mathrm{td}(13.9,6.4)\end{array}$ & $1.84 \mathrm{~m} ; 1.46 \mathrm{~m}$ \\
\hline 3 & & & & & 4.83 brs \\
\hline 4 & & & & & $1.30 \mathrm{~m}$ \\
\hline 5 & $1.71 \mathrm{dd}(12.4,3.4)$ & $1.71 \mathrm{dd}(12.4,3.1)$ & $1.67^{\mathrm{ov}}$ & $1.69 \mathrm{dd}(6.2,2.5)$ & \\
\hline 6 & $1.07 \mathrm{~m} ; 1.50 \mathrm{~m}$ & $1.11^{\mathrm{ov}} ; 1.51^{\mathrm{ov}}$ & $1.07 \mathrm{~m} ; 1.51^{\mathrm{ov}}$ & $1.07 \mathrm{~m} ; 1.50^{\mathrm{ov}}$ & $1.70 \mathrm{~m} ; 0.96^{\mathrm{ov}}$ \\
\hline 7 & $1.52 \mathrm{~m} ; 1.19 \mathrm{~m}$ & $1.22 \mathrm{~m} ; 1.51^{\mathrm{ov}}$ & $0.98 \mathrm{~m} ; 1.50^{\mathrm{ov}}$ & $0.97 \mathrm{~m} ; 1.50^{\mathrm{ov}}$ & $1.52 \mathrm{~m} ; 1.32^{\mathrm{ov}}$ \\
\hline 8 & 1.84 brd (5.1) & 1.83 brd $(6.4)$ & $1.86^{\mathrm{ov}}$ & $1.87 \mathrm{dd}(10.4,3.7)$ & $1.48 \mathrm{~m}$ \\
\hline \multicolumn{6}{|l|}{9} \\
\hline 10 & & & & & 0.82 brd (6.3) \\
\hline 11 & $1.38 \mathrm{~m} ; 2.26 \mathrm{~m}$ & $\begin{array}{c}1.36 \text { brdd }(10.0,3.2) ; \\
2.29 \mathrm{~m}\end{array}$ & $1.38 \mathrm{~m} ; 2.24 \mathrm{~m}$ & $1.40^{\mathrm{ov}} ; 2.28 \mathrm{~m}$ & $1.60 \mathrm{~m} ; 1.24 \mathrm{~m}$ \\
\hline 12 & $\begin{array}{c}1.76 \text { brdd }(11.6,7.5) ; \\
1.86 \mathrm{~m}\end{array}$ & $\begin{array}{c}1.76 \mathrm{~m} ; \\
1.89 \operatorname{brdd}(12.6,3.2)\end{array}$ & $1.58 \mathrm{~m} ; 1.88^{\mathrm{ov}}$ & $1.56 \mathrm{~m} ; 1.92 \mathrm{~m}$ & $1.42 \mathrm{~m} ; 2.22 \mathrm{~m}$ \\
\hline \multicolumn{6}{|l|}{13} \\
\hline \multicolumn{6}{|l|}{14} \\
\hline 15 & $2.16 \mathrm{~m} ; 1.27 \mathrm{~m}$ & $2.13 \mathrm{~m} ; 1.27 \mathrm{~m}$ & $2.15^{\mathrm{ov}} ; 1.32 \mathrm{~m}$ & $2.16 \mathrm{~m} ; 1.32 \mathrm{~m}$ & $\begin{array}{c}2.59 \text { brdd }(13.1,5.3) ; \\
1.21 \mathrm{~m}\end{array}$ \\
\hline 16 & $1.34 \mathrm{~m} ; 2.04 \mathrm{~m}$ & $1.30 \mathrm{~m} ; 2.05 \mathrm{~m}$ & $1.44 \mathrm{~m} ; 2.14^{\mathrm{ov}}$ & $1.43 \mathrm{~m} ; 2.14 \mathrm{~m}$ & $1.68 \mathrm{~m} ; 1.32^{\mathrm{ov}}$ \\
\hline 17 & $1.50 \mathrm{~m}$ & $1.53^{\mathrm{ov}}$ & $1.40 \mathrm{~m}$ & $1.42 \mathrm{~m}$ & \\
\hline 18 & $1.15 \mathrm{~s}$ & $1.15 \mathrm{~s}$ & $1.16^{\mathrm{ov}}$ & $1.15^{\mathrm{ov}}$ & $1.75 \mathrm{~m}$ \\
\hline 19 & $\begin{array}{l}0.56 \mathrm{~d}(4.3) \\
0.91^{\mathrm{ov}}\end{array}$ & $\begin{array}{l}0.56 \mathrm{~d}(4.2) \\
\quad 0.91^{\mathrm{ov}}\end{array}$ & $\begin{array}{l}0.57 \mathrm{~d}(4.0) \\
\quad 0.92^{\mathrm{ov}}\end{array}$ & $\begin{array}{l}0.56 \mathrm{~d}(4.4) \\
\quad 0.92^{\mathrm{ov}}\end{array}$ & $2.65^{\mathrm{ov}}(2 \mathrm{H})$ \\
\hline 20 & $1.54 \mathrm{~m}$ & $1.53^{\mathrm{ov}}$ & $1.51^{\mathrm{ov}}$ & $1.52^{\mathrm{ov}}$ & \\
\hline 21 & $0.91^{\mathrm{ov}}$ & $0.91^{\mathrm{ov}}$ & $0.91^{\mathrm{ov}}$ & $0.91^{\mathrm{ov}}$ & 5.30 brd (3.7) \\
\hline 22 & $1.04 \mathrm{~m} ; 1.42 \mathrm{~m}$ & $1.05 \mathrm{~m} ; 1.43 \mathrm{~m}$ & $1.30 \mathrm{~m} ; 1.50^{\mathrm{ov}}$ & $1.28 \mathrm{~m} ; 1.51^{\mathrm{ov}}$ & $\begin{array}{c}1.17 \mathrm{~m} ; \\
2.41 \mathrm{dd}(15.1,5.1)\end{array}$ \\
\hline 23 & $1.60 \mathrm{~m} ; 1.57 \mathrm{~m}$ & $1.63 \mathrm{~m} ; 1.59 \mathrm{~m}$ & $1.35 \mathrm{~m} ; 1.50^{\mathrm{ov}}$ & $1.40^{\mathrm{ov}}(2 \mathrm{H})$ & $0.77 \mathrm{~d}(7.0)$ \\
\hline 24 & $5.27 \mathrm{t}(6.4)$ & $4.78 \mathrm{dd}(10.2,1.9)$ & 3.22 brd (10.4) & $2.88 \mathrm{dd}(8.8,1.9)$ & $0.90 \mathrm{~s}$ \\
\hline 25 & & & & & $0.93 \mathrm{~s}$ \\
\hline 26 & $1.47 \mathrm{~s}$ & $1.16^{\mathrm{ov}}$ & $1.16^{\mathrm{ov}}$ & $1.16^{\mathrm{ov}}$ & $0.97 \mathrm{~s}$ \\
\hline 27 & $1.42 \mathrm{~s}$ & $1.16^{\mathrm{ov}}$ & $1.13 \mathrm{~s}$ & $1.12 \mathrm{~s}$ & \\
\hline 28 & $1.01 \mathrm{~s}$ & $1.01 \mathrm{~s}$ & $1.01 \mathrm{~s}$ & $1.01 \mathrm{~s}$ & $1.20 \mathrm{~s}$ \\
\hline 29 & $1.12 \mathrm{~s}$ & $1.12^{\mathrm{ov}}$ & $1.11 \mathrm{~s}$ & $1.11 \mathrm{~s}$ & 5.00 brs; 4.92 brs \\
\hline \multicolumn{6}{|l|}{30} \\
\hline $24-\mathrm{OCOCH}_{3}$ & $2.08 \mathrm{~s}$ & $2.08 \mathrm{~s}$ & & & \\
\hline $25-\mathrm{OCOCH}_{3}$ & $1.94 \mathrm{~s}$ & & & & \\
\hline 24-OMe & & & & $3.49 \mathrm{~s}$ & \\
\hline $30-\mathrm{OMe}$ & & & $3.64 \mathrm{~s}$ & $3.64 \mathrm{~s}$ & \\
\hline $3-\mathrm{OCOCH}_{3}$ & & & & & $2.02 \mathrm{~s}$ \\
\hline $21-\mathrm{OCOCH}_{3}$ & & & & & $1.99 \mathrm{~s}$ \\
\hline
\end{tabular}

${ }^{\text {ov } O v e r l a p p e d ~ s i g n a l s ~ w i t h i n ~ a ~ c o l u m n ; ~}{ }^{*}$ Recorded with a $400 \mathrm{MHz}$ instrument; ${ }^{* *}$ Recorded with a $500 \mathrm{MHz}$ instrument. 
Table $2 .{ }^{13} \mathrm{C}-\mathrm{NMR}$ data of compounds $1 \mathrm{a}-\mathbf{1 d}$ and $\mathbf{5 a}$.

\begin{tabular}{|c|c|c|c|c|c|}
\hline Position & $\begin{array}{c}1 \mathrm{a}^{\star} \\
\text { (in } \mathrm{MeOD} \text { ) }\end{array}$ & $\begin{array}{c}1 b^{*} \\
\text { (in } \mathrm{MeOD} \text { ) }\end{array}$ & $\begin{array}{c}1 c^{* *} \\
\text { (in } \mathrm{MeOD} \text { ) }\end{array}$ & $\begin{array}{c}1 \mathrm{~d}^{* *} \\
\text { (in } \mathrm{MeOD} \text { ) }\end{array}$ & $\begin{array}{c}5 \mathrm{a}^{*} \\
\text { (in } \mathrm{CDCl}_{3}-\mathrm{MeOD} \text { ) }\end{array}$ \\
\hline 1 & 34.6 & 34.6 & 34.7 & 34.6 & 16.9 \\
\hline 2 & 38.2 & 38.2 & 38.2 & 38.2 & 32.6 \\
\hline 3 & 219.2 & 219.3 & 219.0 & 219.1 & 75.7 \\
\hline 4 & 51.1 & 51.1 & 51.1 & 51.1 & 48.5 \\
\hline 5 & 49.3 & 49.3 & 49.4 & 49.4 & 37.6 \\
\hline 6 & 21.9 & 21.9 & 21.9 & 21.9 & 41.8 \\
\hline 7 & 27.9 & 27.9 & 28.1 & 28.1 & 18.0 \\
\hline 8 & 46.8 & 46.8 & 47.0 & 46.9 & 52.3 \\
\hline 9 & 21.7 & 21.7 & 21.6 & 21.6 & 38.2 \\
\hline 10 & 28.5 & 28.5 & 28.5 & 28.6 & 61.7 \\
\hline 11 & 29.4 & 29.4 & 29.4 & 29.4 & 39.0 \\
\hline 12 & 34.9 & 34.9 & 35.0 & 35.0 & 27.7 \\
\hline 13 & 48.5 & 48.5 & 48.9 & 48.9 & 52.5 \\
\hline 14 & 63.8 & 63.8 & 64.4 & 64.4 & 41.5 \\
\hline 15 & 32.6 & 32.6 & 32.5 & 32.4 & 28.7 \\
\hline 16 & 30.4 & 30.4 & 30.6 & 30.5 & 37.0 \\
\hline 17 & 53.6 & 53.6 & 54.1 & 53.8 & 31.9 \\
\hline 18 & 18.4 & 18.5 & 18.3 & 18.3 & 44.1 \\
\hline 19 & 30.6 & 30.6 & 30.8 & 30.8 & 27.1 \\
\hline 20 & 35.8 & 35.9 & 36.6 & 36.8 & 141.9 \\
\hline 21 & 18.5 & 18.6 & 18.8 & 18.8 & 74.9 \\
\hline 22 & 33.0 & 33.3 & 34.3 & 34.5 & 40.1 \\
\hline 23 & 26.4 & 26.6 & 28.7 & 28.5 & 11.6 \\
\hline 24 & 77.8 & 80.7 & 78.6 & 90.7 & 16.1 \\
\hline 25 & 84.2 & 72.8 & 73.9 & 74.4 & 20.3 \\
\hline 26 & 22.7 & 25.6 & 25.8 & 25.8 & 18.3 \\
\hline 27 & 22.5 & 25.9 & 24.8 & 25.7 & 179.3 \\
\hline 28 & 22.6 & 22.7 & 22.6 & 22.6 & 33.9 \\
\hline 29 & 21.4 & 21.4 & 21.4 & 21.4 & 117.1 \\
\hline 30 & 180.1 & 180.1 & 178.4 & 178.4 & \\
\hline 24- $\mathrm{O}_{-} \mathrm{COCH}_{3}$ & 172.5 & 172.9 & & & \\
\hline $24-\mathrm{OCO}_{\mathbf{C H}}$ & 20.9 & 21.1 & & & \\
\hline $25-\mathrm{OCOCH}_{3}$ & 172.1 & & & & \\
\hline $25-\mathrm{OCO}^{\mathrm{C}} \mathrm{H}_{3}$ & 22.2 & & & & \\
\hline 24-OMe & & & & 61.4 & \\
\hline 30-OMe & & & 51.5 & 51.5 & \\
\hline
\end{tabular}




\begin{tabular}{lc} 
Continued & \\
\hline $3-\mathrm{OCOCH}_{3}$ & 21.5 \\
$3-\mathrm{OCOCH}_{3}$ & 172.4 \\
$21-\mathrm{OCOCH}_{3}$ & 21.8 \\
$21-\mathrm{OCOCH}_{3}$ & 171.8 \\
\hline
\end{tabular}

${ }^{*}$ Recorded with a $400 \mathrm{MHz}$ instrument; ${ }^{* *}$ Recorded with a $500 \mathrm{MHz}$ instrument.

\subsubsection{Methylation of Glaucatarnoic Acid A (1)}

Compound 1 (50 mg, $102.4 \mathrm{mmol}$ ) was dissolved in DMF (5 mL) and dry $\mathrm{NaH}$ (2.5 mg) was added, followed by MeI (25 mg) (Scheme 2). The mixture was allowed to reflux over a water bath at $60^{\circ} \mathrm{C}$ for 24 hours. The solvent was distilled off and the residue poured into water. The oil formed from the reaction mixture was extracted with EtOAc, then washed and dried. Evaporation of the organic solvent left a residue which was separated over a column of silica gel ( $n$-Hex/ $\left.\mathrm{Me}_{2} \mathrm{CO}, 9: 1\right)$ to yield glaucartanoic acid A methyl ester (1c, $5.2 \mathrm{mg}, 10 \%$ ) and 24-methoxyglaucartanoic acid A methyl ester (1d, $26.7 \mathrm{mg}, 51 \%$ ).

Glaucartanoic acid A methyl ester (1c): White powder (from $\mathrm{Me}_{2} \mathrm{CO}$ ); $[\alpha]_{\mathrm{D}}^{23}-17.7(c 0.10, \mathrm{MeOH}) ;{ }^{1} \mathrm{H}\left(500 \mathrm{MHz}, \mathrm{CD}_{3} \mathrm{OD}\right)$ and ${ }^{13} \mathrm{C}(125 \mathrm{MHz}$ $\left.\mathrm{CD}_{3} \mathrm{OD}\right)$ NMR data, see Table 1 and Table 2 respectively; positive ESIMS m/z (rel. int.) $503[\mathrm{M}+\mathrm{H}]^{+}(6), 525[\mathrm{M}+\mathrm{Na}]^{+}(100)$; HRESIMS m/z $503.3733[\mathrm{M}+$ $\mathrm{H}]^{+}$(calcd for $\mathrm{C}_{31} \mathrm{H}_{51} \mathrm{O}_{5}, 503.3736$ ).

24-methoxyglaucartanoic acid A methyl ester (1d): White powder (from $\left.\mathrm{Me}_{2} \mathrm{CO}\right) ;[\alpha]_{\mathrm{D}}^{23}-29.7$ (c 0.10, $\left.\mathrm{MeOH}\right) ;{ }^{1} \mathrm{H}\left(500 \mathrm{MHz}, \mathrm{CD}_{3} \mathrm{OD}\right)$ and ${ }^{13} \mathrm{C}(125$ $\left.\mathrm{MHz}, \mathrm{CD}_{3} \mathrm{OD}\right) \mathrm{NMR}$ data, see Table 1 and Table 2 respectively; positive ESIMS $\mathrm{m} / \mathrm{z}$ (rel. int.) $517[\mathrm{M}+\mathrm{H}]^{+}(7), 539[\mathrm{M}+\mathrm{Na}]^{+}(70), 540[\mathrm{M}+\mathrm{H}+\mathrm{Na}]^{+}$(12); HRESIMS m/z 517.3896 $[\mathrm{M}+\mathrm{H}]^{+}\left(\right.$calcd for $\left.\mathrm{C}_{32} \mathrm{H}_{53} \mathrm{O}_{5}, 517.3893\right)$.

\subsubsection{Acetylation of 3 $\beta, 21 \beta$-Dihydroxy-30-Nor-(D:A)-Friedoolean-20}

(29)-En-27-Oic Acid (5)

Compound 5 (10 mg, $21.83 \mathrm{mmol}$ ) was treated at room temperature as described above for 1 and afforded the diacetyl derivative $5 \mathrm{a}(8.9 \mathrm{mg}, 76 \%)$ (Scheme 3 ).

$3 \beta, 21 \beta$-diacetoxy-30-nor-(D:A)-friedoolean-20(29)-en-27-oic acid (5a): White powder (from $\mathrm{Me}_{2} \mathrm{CO}$ ); $[\alpha]_{\mathrm{D}}^{23}+18.4$ (c $0.10, \mathrm{CHCl}_{3}$ ); ${ }^{1} \mathrm{H}(400 \mathrm{MHz}$, $\left.\mathrm{CDCl}_{3}-\mathrm{CD}_{3} \mathrm{OD}\right)$ and ${ }^{13} \mathrm{C}\left(100 \mathrm{MHz}, \mathrm{CDCl}_{3}-\mathrm{CD}_{3} \mathrm{OD}\right) \mathrm{NMR}$ data, see Table 1 and Table 2 respectively; positive ESIMS m/z (rel. int.) $565[\mathrm{M}+\mathrm{Na}]^{+}(50), 483[\mathrm{M}$ $-\mathrm{OAc}^{+}$(100), $423[\mathrm{M}-\mathrm{H}-2 \mathrm{OAc}]^{+}(12) ; \mathrm{HRESIMS} \mathrm{m/z} 565.3503[\mathrm{M}+\mathrm{Na}]^{+}$ (calcd for $\mathrm{C}_{33} \mathrm{H}_{50} \mathrm{O}_{6} \mathrm{Na}, 565.3505$ ).

\subsection{Chromatography Methods}

Column chromatography was performed on silica gel G (100 - 200 and $300-400$ mesh, Qingdao Haiyang Chemical Co., Ltd., People's Republic of China) and Sephadex LH-20 (40 - $70 \mu \mathrm{m}$, Amersham Pharmacia Biotech AB, Sweden). TLC was carried out on precoated silica gel plates (Qingdao Haiyang Chemical Co.), and spots were visualized by heating the plates at $90^{\circ} \mathrm{C}$ after they were dipped into a $10 \%$ ethanolic $\mathrm{H}_{2} \mathrm{SO}_{4}$ solution. Solvents were distilled prior to use. 


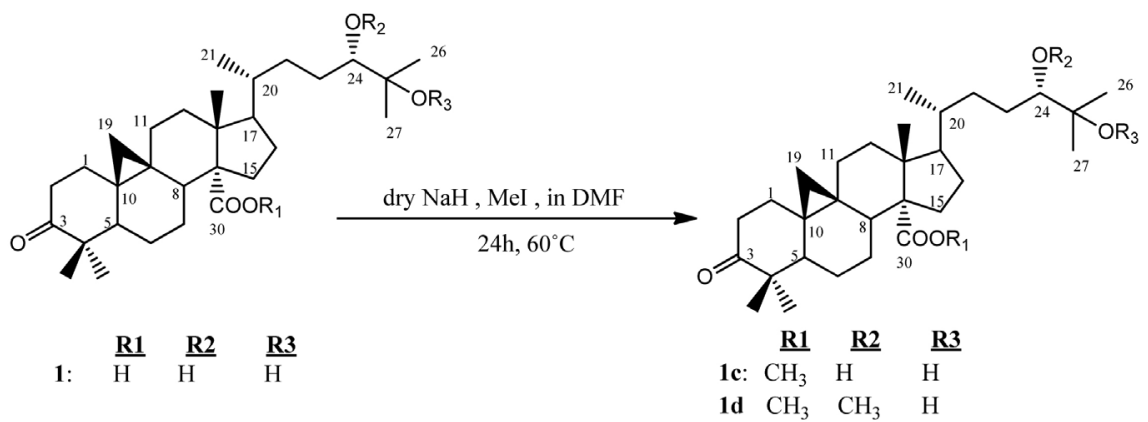

Scheme 2. Semisynthesis of $1 \mathrm{c}$ and $\mathbf{1 d}$ from glaucartanoic acid A (1).

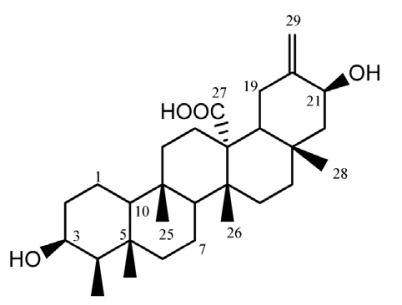

5

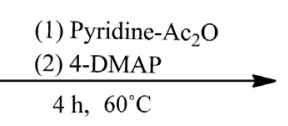

(2) 4-DMAP $60^{\circ} \mathrm{C}$



5a

Scheme 3. Semisynthesis of 5a from 3 $\beta, 21 \beta$-dihydroxy-30-nor-(D:A)-friedoolean-20 (29)-en-27-oic acid (5).

\subsection{Analytical Analyses}

Optical rotations were measured with a JASCO P-1020 digital polarimeter. The NMR spectra were recorded on Bruker AV-400 or DRX-500 NMR spectrometers. Chemical shifts $(\delta)$ are expressed in ppm with reference to TMS, and coupling constants $(/)$ are given in Hz. ESIMS and HRESIMS were carried out on an API Qstar time-of-flight spectrometer.

\subsection{Antimicrobial Assay}

\subsubsection{Microorganisms and Growth Conditions}

The studied microorganisms consisted of sensitive and multidrug resistant Grampositive bacteria (Staphylococcus aureus ATCC25923, methicillin sensitive $S$. aureus MSSA1, methicillin resistant $\mathcal{S}$. aureus MRSA3, methicillin resistant $\mathcal{S}$. aureus MRSA4), Gram-negative bacteria (Shigella flexneri SDINT, Pseudomonas aeruginosa ATCC27853) and three strains of yeasts (Candida tropicalis PK233, Candida albicans ATCC10231 and Cryptococcus neoformans H99) taken from our laboratory collection. The bacterial and fungal species were grown at $37^{\circ} \mathrm{C}$ and maintained on nutrient agar (NA, Conda, Madrid, Spain) and Sabouraud Dextrose Agar (SDA, Conda) slants respectively.

\subsubsection{Determination of Minimum Inhibitory Concentration (MIC) and Minimum Microbicidal Concentration (MMC)}

INT colorimetric assay [23] was performed to assess the minimal inhibitory concentrations (MICs) of crude extract and compounds against a panel of yeasts, Gram-negative and Gram-positive bacteria. Briefly, test samples were first dissolved in dimethyl sulfoxide (DMSO). The solution obtained was then added to 
Mueller Hinton Broth (MHB) for bacteria or Sabouraud Dextrose Broth (SDB) for yeasts and serially diluted twofold (in a 96-well microplate). One hundred microlitres $(100 \mu \mathrm{L})$ of inoculum $\left(1.5 \times 10^{6} \mathrm{CFU} / \mathrm{mL}\right.$ for bacteria and $10^{5}$ spores/ $\mathrm{mL}$ for yeasts) prepared in $\mathrm{MHB} / \mathrm{SDB}$ was added. The plates were covered and agitated to mix the contents of the wells using a plate shaker and incubated at $35^{\circ} \mathrm{C}$ for $24 \mathrm{~h}$ (for bacteria) or for $48 \mathrm{~h}$ (for yeasts). The final concentration of DMSO was $1 \%$ and does not affect the microbial growth. Wells containing $\mathrm{MHB} / \mathrm{SDB}, 100 \mu \mathrm{L}$ of inoculum, and DMSO at a final concentration of $1 \%$ served as a negative control. Ciprofloxacin (Sigma-Aldrich, Steinheim, Germany) and nystatin (Merck, Darmstadt, Germany) were used as reference drugs for bacteria and yeasts respectively. The MIC values of samples were determined by adding $40 \mu \mathrm{L}$ of a $0.2 \mathrm{mg} / \mathrm{mL} p$-iodonitrotetrazolium violet solution followed by incubation at $35^{\circ} \mathrm{C}$ for $30 \mathrm{~min}$. Viable microorganisms reduced the colourless dye to pink. MIC was defined as the lowest sample concentrations that prevented this change and exhibited complete inhibition of microbial growth. All assays were performed in triplicate and repeated thrice. For the determination of MMC values, a portion of liquid $(5 \mu \mathrm{L})$ from each well that showed no growth of microorganism was plated on Mueller Hinton Agar or SDA and incubated at $35^{\circ} \mathrm{C}$ for $24 \mathrm{~h}$ (for bacteria) or $35^{\circ} \mathrm{C}$ for $48 \mathrm{~h}$ (for yeasts). The lowest concentrations that yielded no growth after these subcultures were taken as the MMC values.

\section{Results and Discussion}

\subsection{Chemical Analysis}

The $\mathrm{MeOH}$ extract from the leaves of $C$. glauca was subjected to column chromatography (CC) over silica gel to afford nine known compounds including glaucartanoic acid A (1: $\mathrm{C}_{30} \mathrm{H}_{48} \mathrm{O}_{5} ; \mathrm{m} / \mathrm{z}$ 488) [11], caloncobalactone B $\left(2: \mathrm{C}_{30} \mathrm{H}_{48} \mathrm{O}_{6}\right.$; $\mathrm{m} / \mathrm{z}$ 504) [12], glaucalactone (3: $\left.\mathrm{C}_{29} \mathrm{H}_{44} \mathrm{O}_{4} ; \mathrm{m} / \mathrm{z} 456\right)$ [12], glaucartanoic acid B (4: $\mathrm{C}_{30} \mathrm{H}_{46} \mathrm{O}_{5} ; \mathrm{m} / \mathrm{z}$ 486) [11], 3 $\beta, 21 \beta$-dihydroxy-30-nor-(D:A)-friedoolean-20(29)-en27-oic acid $\left(5: \mathrm{C}_{29} \mathrm{H}_{46} \mathrm{O}_{4} ; \mathrm{m} / \mathrm{z} 458\right)$ [8], caloncobic acid A $\left(6: \mathrm{C}_{30} \mathrm{H}_{46} \mathrm{O}_{4} ; \mathrm{m} / \mathrm{z} 470\right)$ [12], caloncobic acid C (7: $\mathrm{C}_{30} \mathrm{H}_{48} \mathrm{O}_{6} ; \mathrm{m} / \mathrm{z}$ 504) [13], caloncobalactone $\mathrm{C}$ (8: $\mathrm{C}_{33} \mathrm{H}_{52} \mathrm{O}_{5} ; \mathrm{m} / \mathrm{z}$ 528) [13] and glaucalactone $\mathrm{B}\left(9: \mathrm{C}_{29} \mathrm{H}_{46} \mathrm{O}_{4} ; \mathrm{m} / \mathrm{z}\right.$ 458) [13]. Chemical derivatizations including acetylation and methylation of compound 1 and acetylation of compound 5 respectively, afforded five previously unreported semisynthetic derivatives $(\mathbf{1} \mathrm{a}-\mathbf{1} \mathbf{b}$ and $\mathbf{5 a}$ ). The structures of these compounds (Figure 1) were elucidated by the means of $1 \mathrm{D}$ and $2 \mathrm{D}$ NMR spectroscopy, MS and comparison of these data with those reported in the literature.

Compound 1a, obtained from the acetylation reaction of 1 (Scheme 1), was isolated as a white powder in acetone. The molecular formula $\mathrm{C}_{34} \mathrm{H}_{52} \mathrm{O}_{7}$ was deduced from its HR-ESI-MS which displayed the pseudo-molecular ion peak at $\mathrm{m} / \mathrm{z} 595.3612[\mathrm{M}+\mathrm{Na}]^{+}$. This mass was 84 mass units higher than that of 1 , indicating the addition of two acetyl units as compared to 1 . The positive ion mode ESI-MS of 1a also confirmed the presence of two acetoxy groups in the molecule by displaying the ion peaks at $\mathrm{m} / \mathrm{z} 513[\mathrm{M}-\mathrm{OAc}]^{+}$and $453[\mathrm{M}-\mathrm{H}-2 \mathrm{OAc}]^{+}$. 


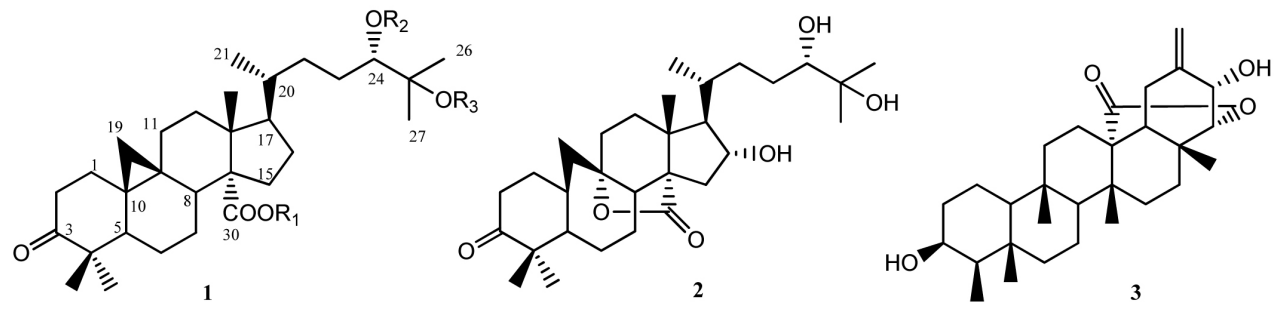

$\begin{array}{llll} & \underline{\mathbf{R 1}} & \underline{\mathbf{R 2}} & \underline{\mathbf{R 3}} \\ \text { 1: } & \mathrm{H} & \mathrm{H} & \mathrm{H} \\ \text { 1a: } & \mathrm{H} & \mathrm{Ac} & \mathrm{Ac} \\ \text { 1b: } & \mathrm{H} & \mathrm{Ac} & \mathrm{H} \\ \text { 1c: } & \mathrm{CH}_{3} & \mathrm{H} & \mathrm{H} \\ \text { 1d } & \mathrm{CH}_{3} & \mathrm{CH}_{3} & \mathrm{H}\end{array}$

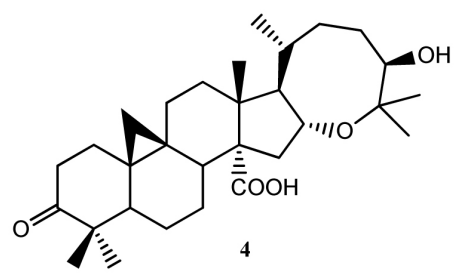


$\begin{array}{lclr} & \underline{\mathbf{R} 1} & \underline{\mathbf{R 2}} & \underline{\mathbf{R} 3} \\ \text { 5: } & \mathrm{H} & \mathrm{H} & \mathrm{H} \\ \text { 5a: } & \mathrm{Ac} & \mathrm{H} & \mathrm{Ac}\end{array}$<smiles>C=C(C)[C@H](O)CC[C@H](C)[C@H]1CC[C@]2(C(=O)O)[C@@H]3CCC4C(C)(C)C(=O)CC[C@]4(C)[C@]3(C)CC[C@]12C</smiles>

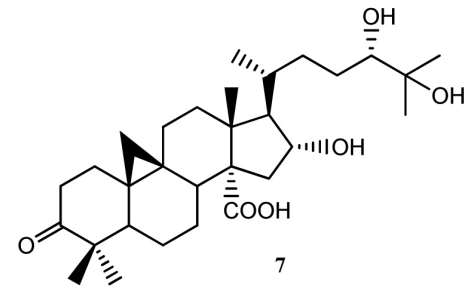
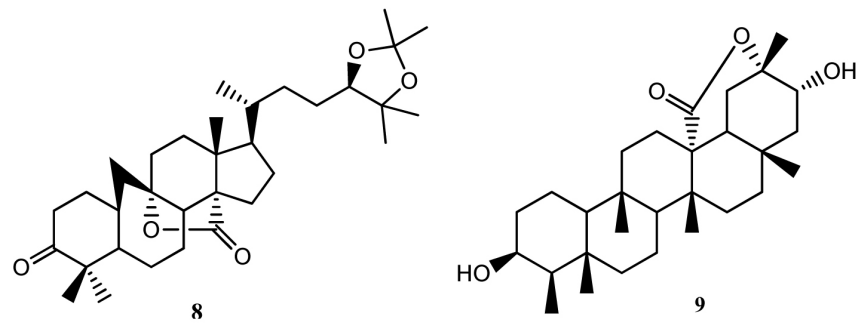

Figure 1. Structures of isolated compounds (1 - 9) from the leaves of Caloncoba glauca and semisynthetic derivatives $(\mathbf{1} \mathrm{a}-\mathbf{1} \mathrm{d}$ and $\mathbf{5 a})$.

The NMR data of 1a revealed additional signals compared to those of glaucartanoic acid $\mathrm{A}(1)$ at $\delta_{\mathrm{H}} 2.08$ and 1.94 (each $3 \mathrm{H}, \mathrm{s}$ ) in the ${ }^{1} \mathrm{H}$-NMR spectrum (Table 1) and at $\delta_{\mathrm{C}} 172.5,172.1,22.2$ and 20.9 in the ${ }^{13} \mathrm{C}$-NMR spectrum (Table 2) assignable to two acetoxy groups. The downfield shift of the $\mathrm{H}-24$ proton signal from $\delta_{\mathrm{H}} 3.21$ (in compound 1) to $\delta_{\mathrm{H}} 5.27$ (in 1a) and of C-25 carbon signal from $\delta_{\mathrm{C}} 73.0$ (in compound 1) to $\delta_{\mathrm{C}} 84.2$ (in 1a) indicated the locations of the acetoxy groups at C-24 and C-25 respectively. Compound $1 \mathrm{a}$ was thus elucidated as $24(S)$, 25-diacetoxy-3-oxocycloartan-30-oic acid and trivially named diacetylglaucartanoic acid $\mathrm{A}$.

Compound $1 \mathrm{~b}$, obtained from the acetylation reaction of 1 (Scheme 1), was isolated as a white powder in acetone. Its molecular formula was determined as $\mathrm{C}_{32} \mathrm{H}_{50} \mathrm{O}_{6}$ from the pseudo-molecular ion peak observed in its HR-ESI-MS at $\mathrm{m} / \mathrm{z}$ $553.3505[\mathrm{M}+\mathrm{Na}]^{+}$. This mass was 42 mass units higher than that of 1 , corres- 
ponding to the addition of one acetyl unit. The presence of a remaining free hydroxyl group in $\mathbf{1 b}$ was further confirmed by the base peak observed at $\mathrm{m} / \mathrm{z} 513$ $\left[\mathrm{M}-\mathrm{H}_{2} \mathrm{O}+\mathrm{H}\right]^{+}$in its positive ion mode ESI-MS. Its ${ }^{1} \mathrm{H}$ and ${ }^{13} \mathrm{C}$ NMR data (Table 1 and Table 2) compared to those of compound 1 revealed additional signals at $\delta_{\mathrm{H}} 2.08(3 \mathrm{H}, \mathrm{s})$ and $\delta_{\mathrm{C}} 172.9$ and 21.1 assignable to the acetoxy group. The downfield shift of the H-24 proton signal from $\delta_{\mathrm{H}} 3.21$ (in 1) to $\delta_{\mathrm{H}} 4.78$ (in $1 \mathrm{~b}$ ) together with the deshielding of the C-24 carbon signal at $\delta_{\mathrm{C}} 80.8$ (instead of $\delta_{\mathrm{C}} 78.6$ in 1 ) indicated the location of the acetoxy group at C-24. This was further confirmed by the $\mathrm{HMBC}$ correlation of $\mathrm{H}-24$ with the carbonyl carbon at $\delta_{\mathrm{C}}$ 172.9. Compound $1 \mathrm{~b}$ was thus elucidated as a new semi-synthetic derivative named 24-acetylglaucartanoic acid A.

Compound $1 \mathrm{c}$ was obtained from the methylation reaction of 1 (Scheme 2), and precipitated as a white powder in acetone. Its HR-ESI-MS displayed a pseudo-molecular ion peak at $\mathrm{m} / \mathrm{z} 525.3573[\mathrm{M}+\mathrm{Na}]^{+}$compatible with the molecular formula $\mathrm{C}_{31} \mathrm{H}_{50} \mathrm{O}_{5}$, indicating an additional methyl group as compared to compound 1. Indeed, the only difference between the NMR data of compound 1c (Table 1 and Table 2) and those of compound 1 was the appearance of signals attributable to a methoxyl group in the ${ }^{1} \mathrm{H}$ and ${ }^{13} \mathrm{C}$ NMR spectra of $1 \mathrm{c}$ at $\delta_{\mathrm{H}} / \delta_{\mathrm{C}} 3.64 / 51.5$. The cross-peak observed in its $\mathrm{HMBC}$ spectrum between the methoxyl protons and the carbonyl carbon at $\delta_{\mathrm{C}} 178.4(\mathrm{C}-30)$ revealed the presence of a methyl ester group. Compound $1 \mathrm{c}$ was thus elucidated as $24(S), 25-$ dihydroxy-3-oxocycloartan-30-carboxylic acid methyl ester and trivially named glaucartanoic acid A methyl ester.

Compound 1d, obtained from the methylation reaction of 1 (Scheme 2), was isolated as a white powder in acetone. Its HR-ESI-MS displayed a pseudo-molecular ion peak at $\mathrm{m} / \mathrm{z} 539.3820[\mathrm{M}+\mathrm{Na}]^{+}$compatible with the molecular formula $\mathrm{C}_{32} \mathrm{H}_{52} \mathrm{O}_{5}$, indicating one more methoxyl than compound $1 \mathrm{c}$. The NMR data of $1 \mathrm{~d}$ (Table 1 and Table 2) confirmed the presence of two methoxyl groups at $\delta_{\mathrm{H}} / \delta_{\mathrm{C}} 3.64 / 51.5$ and 3.49/61.4 respectively. Detailed comparison of its 1D NMR spectra with those of compound 1 revealed the shifting of signals at 24-position at $\delta_{\mathrm{H}} / \delta_{\mathrm{C}} 2.88 / 90.7$ instead of $\delta_{\mathrm{H}} / \delta_{\mathrm{C}} 3.21 / 78.6$ in 1 indicating the location of one of the methoxyl groups at C-24. This was confirmed by the HMBC spectrum where a long-range connectivity was observed between $\mathrm{H}-24\left(\delta_{\mathrm{H}} 2.88\right)$ and the methoxyl carbon at $\delta_{\mathrm{C}} 61.4(24-\mathrm{OMe})$. The HMBC correlation also observed between the second methoxyl signal at $\delta_{\mathrm{H}} 3.64$ and the carbonyl carbon at $\delta_{\mathrm{C}} 178.4$ (C-30) proved the existence of a methyl ester functionality at C-30. Compound $1 \mathrm{~d}$ was thus characterized as $24(S)$-methoxy-25-hydroxy-3-oxocycloartan-30-carboxylic acid methyl ester and was trivially named 24-methoxyglaucartanoic acid A methyl ester.

Compound 5a was obtained from the acetylation reaction of 5 (Scheme 3), and precipitated as a white powder in acetone. Its molecular formula was established as $\mathrm{C}_{33} \mathrm{H}_{50} \mathrm{O}_{6}$ from the HR-ESI-MS displaying the pseudo-molecular ion peak at $\mathrm{m} / \mathrm{z} 565.3503[\mathrm{M}+\mathrm{Na}]^{+}$. This molecular formula in accordance with the pseudo-molecular mass furthermore 84 mass units higher than that of 5 , re- 
vealed the addition of two acetyl units in the reaction product. The NMR data of 5a (Table 1 and Table 2) exhibited additional signals of two methyl groups at $\delta_{\mathrm{H}} / \delta_{\mathrm{C}} 2.02(3 \mathrm{H}, \mathrm{s}) / 21.5$ and $1.99(3 \mathrm{H}, \mathrm{s}) / 21.8$ and for two ester carbonyls at $\delta_{\mathrm{C}}$ 172.4 and 171.8 as compared to 5 . Notable differences between the NMR data of compounds 5 and $5 \mathrm{a}$ also involved the downfield shift of signals at C-3 position from $\delta_{\mathrm{H}} / \delta_{\mathrm{C}} 3.65 / 72.7$ in compound 5 to $\delta_{\mathrm{H}} / \delta_{\mathrm{C}} 4.83 / 75.7$ in compound $5 \mathrm{a}$, and at position C-21 from $\delta_{\mathrm{H}} / \delta_{\mathrm{C}} 4.19 / 71.5$ (in 5) to $\delta_{\mathrm{H}} / \delta_{\mathrm{C}} 5.30 / 74.9$ (in 5a). This implied the location of acetoxy groups at C-3 and C-21 positions in compound $5 \mathrm{a}$ which was further elucidated as $3 \beta, 21 \beta$-diacetoxy-30-nor-(D:A)-friedoolean-20(29)-en-27oic acid.

\subsection{Antimicrobial Activity}

The $\mathrm{MeOH}$ extract, isolated compounds and some new semisynthetic derivatives were evaluated for their antimicrobial activities against 9 microorganisms including four Gram-positive (Staphylococcus aureus ATCC25923, methicillin sensitive $S$. aureus MSSA1, methicillin resistant $S$. aureus MRSA3, methicillin resistant $S$. aureus MRSA4), two Gram-negative (Shigella flexneri SDINT, Pseudomonas aeruginosa) bacteria and three fungal strains (Candida albicans ATCC10231, Candida tropicalis PK233 and Cryptococcus neoformans H99) (Table 3). The $\mathrm{MeOH}$ extract displayed antimicrobial activity towards 9/9 (100\%) of tested bacterial and fungal strains with good activity (MIC $<100 \mu \mathrm{g} / \mathrm{mL}$ ) [24] against Staphylococcus aureus and Shigella flexneri SDINT ATCC25923. Among the phytochemicals, compounds $\mathbf{3}$ and $\mathbf{5}$ were the most active showing moderate activity $(10<\mathrm{MIC} \leq 100 \mu \mathrm{g} / \mathrm{mL})$ [24] against 5/9 (55.55\%) and 6/9 (66.66\%) tested microbial species, respectively. As shown in Table 3, ciprofloxacin and nystatin used as standard drugs were more potent against yeasts, Gram-positive and Gram-negative bacteria than all the tested samples. A microbicidal effect with $\mathrm{MMC} / \mathrm{MIC}$ ratio $\leq 4$ was noted for most of the samples (MeOH extract, 2, 3, 5, $7,8,1 \mathrm{a}, 1 \mathrm{~b}, 1 \mathrm{~d}$ and $5 \mathrm{a}$ ) indicating their lethal effect. In general, Gram-negative bacteria were found to be more sensitive to the tested samples when compared with Gram-positive bacteria whereas the bacterial species were resistant as compared to fungal strains. These variations may be due to genetic differences between the microorganisms. The known antimicrobial mechanisms associated to the group of chemicals to which the isolated compounds belong may explain the antimicrobial potency of the $\mathrm{MeOH}$ extract. Membrane disruption has been suggested as one of the likely mechanisms of action [25] [26]. This might also explain the antimicrobial activities of isolated triterpenoids and semisynthetic derivatives [25] [26]. Although the fungal strains were more sensitive to the tested compounds than the bacteria strains used in this study, the antibacterial activities of the acetylated (1a and $\mathbf{1 b}$ ) and the methylated (1d) derivatives of cycloartane 1 were improved compared to that of the original substrate mostly on Gram-negative strains. Meanwhile the $O$-acetylation at both the C-3 and C-21 positions of friedelane 5 leading to compound $5 \mathrm{a}$ induced a decrease in the antibacterial activity on both Gram-positive and Gram-negative strains, but an 
Table 3. Antimicrobial activity (MIC and MMC in $\mu \mathrm{g} / \mathrm{mL}$ ) of $\mathrm{MeOH}$ extract, isolated compounds, semisynthetic derivatives and reference antimicrobial drugs.

\begin{tabular}{|c|c|c|c|c|c|c|c|c|c|c|}
\hline \multirow[b]{2}{*}{ Extract/Compounds } & \multirow[b]{2}{*}{$\begin{array}{l}\text { Inhibition } \\
\text { Parameters }\end{array}$} & \multicolumn{6}{|c|}{ Bacteria } & \multicolumn{3}{|c|}{ Yeasts } \\
\hline & & $\begin{array}{l}\text { S. aureus } \\
\text { ATCC }\end{array}$ & MSSA1 & MRSA3 & MRSA4 & SF & $P A$ & $\begin{array}{c}C A \\
\text { ATCC10231 }\end{array}$ & $C T$ PK233 & CNH99 \\
\hline \multirow{3}{*}{$\begin{array}{l}\mathrm{MeOH} \\
\text { extract }\end{array}$} & MIC & 64 & 128 & 128 & 128 & 64 & 128 & 2048 & 2048 & 1024 \\
\hline & MMC & 64 & 128 & 256 & 256 & 64 & 128 & 4096 & 4096 & 2048 \\
\hline & $\mathrm{MMC} / \mathrm{MIC}$ & 1 & 1 & 2 & 2 & 1 & 1 & 2 & 2 & 2 \\
\hline \multirow{3}{*}{1} & MIC & $>256$ & $>256$ & $>256$ & $>256$ & $>256$ & $>256$ & 64 & 128 & 32 \\
\hline & MMC & $>256$ & $>256$ & $>256$ & $>256$ & $>256$ & $>256$ & 256 & 256 & 128 \\
\hline & $\mathrm{MMC} / \mathrm{MIC}$ & I & l & l & l & l & l & 4 & 2 & 4 \\
\hline \multirow{3}{*}{2} & MIC & 256 & $>256$ & $>256$ & $>256$ & 256 & 128 & 256 & 128 & 128 \\
\hline & MMC & 256 & $>256$ & $>256$ & $>256$ & 256 & 128 & 256 & 256 & 256 \\
\hline & $\mathrm{MMC} / \mathrm{MIC}$ & 1 & I & I & I & 1 & 1 & 1 & 2 & 2 \\
\hline \multirow{3}{*}{3} & MIC & 32 & 64 & 256 & 256 & 32 & 32 & 256 & 128 & 64 \\
\hline & MMC & 64 & 128 & 256 & 256 & 32 & 32 & 256 & 128 & 64 \\
\hline & MMC/MIC & 2 & 2 & 1 & 1 & 1 & 1 & 1 & 1 & 1 \\
\hline \multirow{3}{*}{4} & MIC & $>256$ & $>256$ & $>256$ & $>256$ & $>256$ & $>256$ & 128 & 128 & 64 \\
\hline & MMC & $>256$ & $>256$ & $>256$ & $>256$ & $>256$ & $>256$ & 256 & 256 & 128 \\
\hline & $\mathrm{MMC} / \mathrm{MIC}$ & I & I & I & I & I & I & 2 & 2 & 2 \\
\hline \multirow{3}{*}{5} & MIC & 32 & 64 & 64 & 64 & 32 & 32 & $>256$ & 128 & 128 \\
\hline & MMC & 32 & 64 & 64 & 64 & 32 & 32 & $>256$ & 128 & 128 \\
\hline & MMC/MIC & 1 & 1 & 1 & 1 & 1 & 1 & I & 1 & 1 \\
\hline \multirow{3}{*}{6} & MIC & 256 & $>256$ & $>256$ & $>256$ & 256 & 256 & 128 & 128 & 64 \\
\hline & MMC & $>256$ & $>256$ & $>256$ & $>256$ & 256 & 256 & 256 & 256 & 256 \\
\hline & $\mathrm{MMC} / \mathrm{MIC}$ & I & I & I & I & 1 & 1 & 2 & 2 & 4 \\
\hline \multirow{3}{*}{7} & MIC & 128 & $>256$ & $>256$ & $>256$ & 64 & 64 & 256 & 128 & 64 \\
\hline & MMC & 256 & $>256$ & $>256$ & $>256$ & 128 & 128 & 256 & 128 & 128 \\
\hline & MMC/MIC & 2 & I & I & I & 2 & 2 & 1 & 1 & 2 \\
\hline \multirow{3}{*}{8} & MIC & 128 & $>256$ & $>256$ & $>256$ & 128 & 64 & 128 & 128 & 64 \\
\hline & MMC & 128 & $>256$ & $>256$ & $>256$ & 256 & 128 & 256 & 256 & 128 \\
\hline & MMC/MIC & 1 & I & I & I & 2 & 2 & 2 & 2 & 2 \\
\hline \multirow{3}{*}{9} & MIC & 128 & $>256$ & $>256$ & $>256$ & 64 & 64 & 256 & 128 & 32 \\
\hline & MMC & $>256$ & $>256$ & $>256$ & $>256$ & 256 & 128 & 256 & 128 & 128 \\
\hline & $\mathrm{MMC} / \mathrm{MIC}$ & I & I & I & I & 4 & 2 & 1 & 1 & 4 \\
\hline \multirow{3}{*}{$1 \mathrm{a}$} & MIC & 128 & $>256$ & $>256$ & $>256$ & 128 & 64 & 256 & 32 & 32 \\
\hline & MMC & 256 & $>256$ & $>256$ & $>256$ & 256 & 256 & 256 & 64 & 64 \\
\hline & $\mathrm{MMC} / \mathrm{MIC}$ & 2 & I & I & I & 2 & 4 & 1 & 2 & 2 \\
\hline
\end{tabular}




\begin{tabular}{|c|c|c|c|c|c|c|c|c|c|c|}
\hline & MIC & 128 & $>256$ & $>256$ & 256 & 64 & 64 & 64 & 128 & 128 \\
\hline \multirow[t]{3}{*}{$1 b$} & MMC & 128 & $>256$ & $>256$ & 256 & 64 & 64 & 256 & 256 & 128 \\
\hline & MMC/MIC & 1 & I & I & 1 & 1 & 1 & 4 & 2 & 1 \\
\hline & MIC & 128 & $>256$ & $>256$ & $>256$ & 128 & 128 & 256 & 256 & 64 \\
\hline \multirow[t]{3}{*}{$1 d$} & MMC & 256 & $>256$ & $>256$ & $>256$ & 256 & 128 & 256 & 256 & 128 \\
\hline & MMC/MIC & 2 & I & I & I & 2 & 1 & 1 & 1 & 2 \\
\hline & MIC & 128 & 256 & 256 & $>256$ & 128 & 64 & 256 & 128 & 64 \\
\hline \multirow[t]{3}{*}{$5 a$} & MMC & 256 & 256 & 256 & $>256$ & 128 & 128 & 256 & 256 & 128 \\
\hline & MMC/MIC & 2 & 1 & 1 & I & 1 & 2 & 1 & 2 & 2 \\
\hline & MIC & 2 & 1 & 1 & 2 & 16 & 2 & 2 & 0.5 & 1 \\
\hline \multirow[t]{2}{*}{$\operatorname{Ref}^{*}$} & MMC & 2 & 1 & 1 & 2 & 16 & 2 & 2 & 1 & 1 \\
\hline & MMC/MIC & 1 & 1 & 1 & 1 & 1 & 1 & 1 & 2 & 1 \\
\hline
\end{tabular}

SA ATCC25923: Staphylococcus aureus ATCC25923; MSSA1: methicillin sensitive S. aureus MSSA1; MRSA3: methicillin resistant S. aureus MRSA3; MRSA4: methicillin resistant $S$. aureus MRSA4; SF: Shigella flexneri; PA: Pseudomonas aeruginosa; CA ATCC10231: Candida albicans ATCC10231; CT PK233: Candida tropicalis PK233; CNH99: Cryptococcus neoformans H99; MIC: minimum inhibitory concentration; MMC: Minimum microbicidal concentration; /: not determined; ${ }^{*}$ : Ciprofloxacin and nystatin were used as reference drugs for bacteria and yeasts respectively.

increase in the antifungal activity. The lower antibacterial activity mainly on MRSA3 and MRSA4 strains observed for compound 3 with respect to its analogue 5 could arise from lactonization of the $\mathrm{C}-27$ carboxylic group in compound 3. The difference in the antimicrobial activity of compounds having the same basic skeleton highlights the contribution of esterified carboxylic acid group and acetyl substituents in influencing the activity of this series of compounds.

\section{Conclusion}

Phytochemical investigation of the $\mathrm{MeOH}$ extract from the leaves of C. glauca led to the isolation of nine known triterpenoids (1-9). Chemical transformations carried out on glaucartanoic acid A (1) and $3 \beta, 21 \beta$-dihydroxy-30-nor-(D:A)friedoolean-20(29)-en-27-oic acid (5) respectively, afforded five new derivatives (1a-1d and 5a). The $\mathrm{MeOH}$ extract displayed antimicrobial activity towards all the tested pathogenic bacterial and fungal strains with good activity (MIC $<100$ $\mu \mathrm{g} / \mathrm{mL}$ ) against Staphylococcus aureus ATCC25923 and Shigella flexneri SDINT. Compounds 3 and 5 belonging to the friedelane type showed the most potent antimicrobial effect. These results could justify the traditional use of $C$. glauca in the treatment of skin diseases caused by some of the tested microorganisms.

\section{Acknowledgements}

We are grateful to the University of Dschang and the Ministry of Higher Education (Cameroon) for financing some consumable items used in this work.

\section{Conflicts of Interest}

The authors declare they have no competing interest. 


\section{References}

[1] Prestinaci, F., Pezzotti, P. and Pantosti, A. (2015) Antimicrobial Resistance: A Global Multifaceted Phenomenon. Pathogens and Global Health, 109, 309-318. https://doi.org/10.1179/2047773215Y.0000000030

[2] Laxminarayan, R. and Heymann, D. (2012) Challenges of Drug Resistance in the Developing World. British Medical Journal, 344, e1567. https://doi.org/10.1136/bmj.e1567

[3] Fischbach, M.A. and Walsh, C.T. (2009) Antibiotics for Emerging Pathogens. Science, 325, 1089-1093. https://doi.org/10.1126/science.1176667

[4] Jain, S.C., Singh, B. and Jain, R. (2001) Antimicrobial Activity of Triterpenoids from Heliotropium ellipticum. Fitoterapia, 72, 666-668. https://doi.org/10.1016/S0367-326X(01)00267-2

[5] Chiozem, D.D., Trinh-Van-Dufat, H., Wansi, J.D., Djama, C.M., Fannang, V.S., Seguin, E., Tillequin, F. and Wandji, J. (2009) New Friedelane Triterpenoids with Antimicrobial Activity from the Stems of Drypetes paxii. Chemical Pharmaceutical Bulletin, 57, 1119-1122. https://doi.org/10.1248/cpb.57.1119

[6] Da Silva, G.J., Pereira, M. and Da Salvador, J. (2017) Synthesis and Antibacterial Activity of Novel Semi-Synthetic Triterpenoids. FASEB Journal, 31, 777.20.

[7] Giner-Pons, R.M., Gray, A.I., Lavaud, C., Massiot, G., Gibbons, S. and Waterman, P.G. (1992) 30-Norfriedelane Triterpenes from the Stem Bark of Caloncoba glauca. Phytochemistry, 31, 223-225. https://doi.org/10.1016/0031-9422(91)83041-I

[8] Giner, R.M., Gray, A.I., Gibbons, S. and Waterman, P.G. (1993) Friedelane Triterpenes from the Stem Bark of Caloncoba glauca. Phytochemistry, 33, 237-239. https://doi.org/10.1016/0031-9422(93)85433-R

[9] Tchuendem, M.H.K., Ayafor, J.F. and Connolly, J.D. (1996) Lophocarpin and 21 $\beta$-Hydroxylophocarpin, Two New 30-Norfriedelane Triterpenes from Caloncoba lophocarpa. Natural Product Letters, 9, 27-32. https://doi.org/10.1080/10575639608043574

[10] Ziegler, H.L., Staerk, D., Christiensen, J., Olsen, C.E., Sittie, A.A. and Jarosweski, J.W. (2002) New Dammarane and Malabaricane Triterpenes from Caloncoba echinata. Journal of Natural Products, 65, 1764-1765. https://doi.org/10.1021/np0201826

[11] Mpetga, J.D.S., Tene, M., Wabo, H.K., Li, S.-F., Kong, L.-M., He, H.-P., Hao, X.-J. and Tane, P. (2012) Cytotoxic Cycloartanes from the Fruits of Caloncoba glauca. Phytochemistry Letters, 5, 183-187. https://doi.org/10.1016/j.phytol.2011.12.006

[12] Mpetga, J.D.S., Shen, Y., Tane, P., Li, S.-F., He, H.-P., Wabo, H.K., Tene, M., Leng, Y. and Hao, X.-J. (2012) Cycloartane and Friedelane Triterpenoids from the Leaves of Caloncoba glauca and Their Evaluation for Inhibition of $11 \beta$-Hydroxysteroid Dehydrogenases. Journal of Natural Products, 75, 599-604.

https://doi.org/10.1021/np200831c

[13] Mpetga, J.D.S., He, H.P., Hao, X.J., Leng, Y. and Tane, P. (2014) Further Cycloartane and Friedelane Triterpenoids from the Leaves of Caloncoba glauca. Phytochemistry Letters, 7, 52-56. https://doi.org/10.1016/j.phytol.2013.09.015

[14] Douanla, P.D., Tchuendem, M.H.K., Tchinda, A.T., Tabopda, T.K., Zofou, D., Cieckiewicz, E., Frederich, M. and Nkengfack, A.E. (2018) Chemical Constituents of the Leaves of Caloncoba welwitschii Gilg. Phytochemistry Letters, 23, 5-8. https://doi.org/10.1016/j.phytol.2017.10.019

[15] Keugwa, C.V.T., Songue, J.L., Tcho, A.T., Waffo, A.F.K., Wansi, J.D. and Ndom, 
J.C. (2018) Welwitschiilactone A, B and C, Three New 30-Norfriedelane Triterpenes from the Roots of Caloncoba welwitschii (Oliv.) Gilg (Achariaceae). Phytochemistry Letters, 27, 15-19. https://doi.org/10.1016/j.phytol.2018.06.011

[16] Burkill, H.M. (1994) The Useful Plants of West Tropical Africa. 2nd Edition, Royal Botanic Gardens, Kew.

[17] Kwon-Chung, K.J. and Bennett, J.E. (1992) Cryptococcosis. In: Kwon-Chung, K.J. and Bennett, J.E., Eds., Medical Mycology, Lea \& Febiger, Philadelphia, 397-446.

[18] Singh, N., Gayowski, T., Wagener, M.M. and Marino, I.R. (1997) Clinical Spectrum of Invasive Cryptococcosis in Liver Transplant Recipients Receiving Tacrolimus. Clinical Transplantation, 11, 66-70.

[19] Dromer, F., Mathoulin, S., Dupont, B., Letenneur, L., Ronin, O. and French Cryptococcosis Study Group (1996) Individual and Environmental Factors Associated with Infections Due to Cryptococcus neoformans Serotype D. Clinical Infectious Diseases, 23, 91-96. https://doi.org/10.1093/clinids/23.1.91

[20] Damen, F., Mpetga, J.D.S., Demgne, O.M.F., Çelik, İ., Wamba, B.E.N., Tapondjou, L.A., Beng, V.P., Levent, S., Kuete, V. and Tene, M. (2020) Roeperone A, a New Tetraoxygenated Xanthone and Other Compounds from the Leaves of Hypericum roeperianum Schimp. (Hypericaceae). Natural Product Research.

https://doi.org/10.1080/14786419.2020.1845674

[21] Nguemo, R.B.T., Njouendou, A.J., Mpetga, J.D.S., Kengne, I.C., Nago, R.D.T., Fobofou, S.A.T., Wessjohann, L.A., Ngouela, A.S. and Tamokou, JDD. (2020) Antimicrobial Activities of Chemical Constituents from the Flowers of Hypericum lanceolatum Lam. (Hypericaceae). International Journal of Applied Microbiology and Biotechnology Research, 8, 66-72.

[22] Nguetsa, L.Z., Mpetga, J.D.S., Bitchagno, G.T.M., Tadjuidje, J.D., Groß, J., Lenta, B.N., Opatz, T., Sewald, N. and Tene, M. (2021) C-28/C-30 Oxidized Cycloartanes from the Teaves and Twigs of Caloncoba dusenii Gilg. Phytochemistry Letters, 43, 145-149. https://doi.org/10.1016/j.phytol.2021.03.026

[23] Tamokou, J.D.D., Kuiate, J.R., Tene, M., Nwemeguela, K.T.J. and Tane, P. (2011) The Antimicrobial Activities of Extract and Compounds Isolated from Brillantaisia lamium. Iranian Journal of Medical Sciences, 36, 24-31.

[24] Tamokou, J.D.D., Mbaveng, T.A and Kuete, V. (2017) Antimicrobial Activities of African Medicinal Spices and Vegetables. In: Medicinal Spices and Vegetables from Africa: Therapeutic Potential against Metabolic Inflammatory Infectious and Systemic Diseases, Academic Press, Cambridge, 207-237. https://doi.org/10.1016/B978-0-12-809286-6.00008-X

[25] Cowan, M.M. (1999) Plant Products as Antimicrobial Agents. Clinical Microbiology Reviews, 12, 564-582. https://doi.org/10.1128/CMR.12.4.564

[26] Arvind, S., Reg, F.C. and Enzo, A.P. (2004) Identification of Antimicrobial Component of an Ethanolic Extract of Australian Medicinal Plant, Eremophila duttonii. Phytotherapy Research, 18, 615-618. https://doi.org/10.1002/ptr.1507

\section{Supplementary Material}

Supporting information for this article has been uploaded as the electronic supplementary material. 